\title{
Sífilis congênita no estado do Pará-Brasil, 2007 a 2016
}

\author{
Congenital syphilis in the state of Pará-Brazil, 2007 the 2016
}

Sifilis congenita en el estado de Pará-Brasil, 2007 a 2016

\begin{abstract}
Luísa Margareth Carneiro da Silva ${ }^{1}$, Rosa Maria Dias ${ }^{1}$, Andrea das Graças Ferreira Frazão ${ }^{1}$, Ana Lúcia da Silva Rezende ${ }^{1 *}$. Fernanda Maria Lima Moura ${ }^{1}$, Eliete da Cunha Araújo ${ }^{1}$, Maria da Conceição Nascimento Pinheiro ${ }^{1}$, Anderson Raiol Rodrigues ${ }^{1}$, Ana Maria Almeida Souza', Paula Valente Leão'1.
\end{abstract}

\section{RESUMO}

Objetivo: Estimar a incidência e fatores associados à sífilis congênita (SC) nas Regiões Geográficas Intermediárias (RGIs) no Estado do Pará. Método: Estudo descritivo, quantitativo, analítico, sobre SC no Pará, utilizando dados do Sistema de Informação de Agravos de Notificação de 2007 a 2016. Resultados: De 2007 a 2016, foram notificados 4710 casos de SC, com aumentos significativos nas RGls Belém e Marabá. Variáveis que se associaram $(p<0,05)$ com a ocorrência de $S C$, escolaridade materna $(p=0,0224)$ e cor da pele não branca $(p<0,0001)$. A RGI Altamira teve maior percentual de mães menores de 20 anos. A realização do pré-natal variou de $65,8 \%$ a $90,1 \%$. O diagnostico no pré-natal foi mais frequente nas RGI de Altamira (49,7\%), Marabá $(49,7 \%)$ e Santarém (49,2\%). Tratamento inadequado das mães e parceiros não tratados prevaleceram nas Regiões Altamira e Marabá, respectivamente. Conclusão: A incidência da SC foi mais elevada em mulheres maiores de 20 anos, em todas RGIs. O tratamento inadequado, ausência de tratamento do parceiro e diagnóstico de sífilis após o parto foram expressivos, justificando a alta incidência da SC.

Palavras-chave: Sífilis congênita, Cuidado pré-natal, Epidemiologia.

\begin{abstract}
Objective: Estimating the incidence and factors associated with congenital syphilis (CS) in the Intermediate Geographical Regions (IGRs) in the State of Pará. Method: A descriptive, quantitative, analytical study on CS in Pará using data from the Notification System from 2007 to 2016. Results: From 2007 to 2016, 4710 cases of CS were reported, with significant increases in Belém and Marabá RGls. Variables that were associated ( $p$ $<0.05)$ with the occurrence of CS, maternal schooling $(p=0.0224)$ and nonwhite skin color $(p<0.0001)$. The IGR Altamira had a higher percentage of mothers under 20 years of age. Prenatal care ranged from $65.8 \%$ to $90.1 \%$. The Diagnosis in prenatal care was more frequent in the IGR of Altamira (49.7\%), Marabá (49.7\%) and Santarém (49.2\%). Inadequate treatment of mothers and untreated partners prevailed in the Altamira and Marabá Regions, respectively. Conclusion: The incidence of CS was higher in women older than 20 years, in all IGRs. The inadequate treatment, absence of treatment of the partner and diagnosis of syphilis after childbirth were expressive, justifying the high incidence of CS.
\end{abstract}

Key words: Congenital syphilis, Prenatal care, Epidemiology.

\section{RESUMEN}

El objetivo de este estudio es analizar la incidencia y factores asociados a la sífilis congénita (SC) en las regiones geográficas intermediarias (RGIs) en el Estado de Pará. Método: Estudio descriptivo, cuantitativo, analítico, sobre SC en Pará, utilizando datos del Sistema de Información de Agravios de Notificación de 2007

1 Universidade Federal do Pará (UFPA), Belém-Pará. *E-mail: ana_luciasr@yahoo.com.br

SUBMETIDO EM: 5/2019 | ACEITO EM: 6/2019 | PUBLICADO EM: 6/2019

REAS/EJCH | Vol. Sup. 24 | e1003 | DOI: https://doi.org/10.25248/reas.e1003.2019 Página 1 de 10 
a 2016. Resultados: De 2007 a 2016, se notificaron 4710 casos de SC, con aumentos significativos en las RGIs Belém y Marabá. Variables que se asociaron $(p<0,05)$ con la ocurrencia de $S C$, escolaridad materna ( $p$ $=0,0224)$ y color de la piel no blanca $(p<0,0001)$. La RGI Altamira tuvo un mayor porcentaje de madres menores de 20 años. La realización del prenatal varió del 65,8\% al 90,1\%. El diagnóstico en el prenatal fue más frecuente en las RGI de Altamira (49,7\%), Marabá (49,7\%) y Santarém (49,2\%). El tratamiento inadecuado de las madres y los socios no tratados prevalecieron en las regiones Altamira y Marabá, respectivamente. Conclusión: La incidencia de SC fue más elevada en mujeres mayores de 20 años, en todas las RGIs. El tratamiento inadecuado, ausencia de tratamiento del compañero y diagnóstico de sífilis después del parto fueron expresivos, justificando la alta incidencia de la SC.

Palabras clave: Sífilis congénita, Cuidado prenatal, Epidemiología.

\section{INTRODUÇÃO}

A sífilis é uma doença curável e exclusiva do ser humano, transmitida pelo Treponema pallidum por meio do contato sexual com lesões infecciosas das membranas mucosas ou da pele escoriada, por transfusão de sangue, ou por transmissão vertical de uma mulher grávida para seu feto (LAFETA KR et al., 2016; WHO, 2018). Quando não tratada, pode levar a resultados fetais funestos no segundo ou terceiro trimestres da gravidez (BRASIL, 2018).

No ano de 2016, no mundo, aproximadamente 1 milhão de mulheres grávidas tiveram infecção por sífilis e estavam em risco de resultados adversos da gravidez, como natimorto e morte neonatal devido à sífilis congênita, com mais de 200 mil mortes fetais e neonatais e pondo em risco de morte prematura mais de 200 mil crianças (WHO, 2019).

No Brasil, ano de 2016, foram notificados 20.474 casos de sífilis congênita. Entretanto, nos últimos seis anos, de 2010 a 2016 o número de casos vem aumentando, no país. A taxa de incidência de sífilis congênita e as taxas de detecção de sífilis em gestante por mil nascidos vivos aumentaram cerca de três vezes nesse período, passando de 2,4 para 6,8 e de 3,5 para 12,4 casos por mil nascidos vivos, respectivamente. Esse aumento ocorreu possivelmente devido ao aumento da cobertura de testagem, com a ampliação do uso de testes rápidos, redução do uso de preservativos, resistência dos profissionais de saúde à administração da penicilina na Atenção Básica, desabastecimento mundial da penicilina. Ainda podendo ser atribuído ao aperfeiçoamento do sistema de vigilância com a efetivação das notificações (BRASIL, 2017).

Não obstante os avanços sobre o diagnóstico e o tratamento da gestante, o controle da sífilis na gestação ainda é um desafio para a atenção ao pré-natal, devido à infecção se apresentar assintomática na gestação (SARACENI V et al., 2017). A estratégia mundial do setor da saúde da OMS contra as infecções de transmissão sexual é acabar no mundo até 2030, com o problema de saúde pública por elas representado, dentre elas: reduzir a incidência do T. pallidum a 90\% em todo o mundo, em comparação a 2018 e reduzir para 50 o número máximo de casos de sífilis congênita por 100.000 nascimentos vivos em $80 \%$ dos países (WHO, 2016).

Considerando que o rastreamento da sífilis durante a gravidez e o tratamento específico são as únicas formas de impedir eficientemente a morte fetal e implantar tais medidas representa benefícios evidentes e irrefutáveis, a presente pesquisa tem como objetivo investigar a incidência de sífilis congênita no Estado do Pará e sua relação com fatores sociodemográficos.

\section{MÉTODOS}

Trata-se de um estudo retrospectivo, descritivo, quantitativo, analítico e documental, sobre sífilis em gestantes e sífilis congênita no Estado do Pará. Utilizaram-se os dados secundários oriundos das fichas de investigação/notificação registradas nos bancos do Sistema de Informação de Agravos de Notificação (SINAN), no período de 2007 a 2016. 
O Estado do Pará está localizado na Região Norte do Brasil, apresenta uma população estimada (2018) de 8.513.497 habitantes, distribuída em uma área de 1.245.759,305 Km², é segundo maior estado da federação, sua área representa $14,7 \%$ do território brasileiro e é constituído por 144 municípios (IBGE, 2019).

Para este estudo foi considerada a divisão territorial e administrativa do Estado, elaborada pelo Instituto Brasileiro de Geografia e Estatística (IBGE); atualizando o quadro regional do País, que dividia os estados em mesorregiões e microrregiões e que nesse estudo recebem os nomes de Regiões Geográficas Intermediárias e Regiões Geográficas Imediatas, respectivamente (IBGE, 2017). Nessa revisão, o IBGE divide o Estado do Pará em sete Regiões Intermediárias: Altamira, Belém, Breves, Castanhal, Marabá, Redenção e Santarém.

As Regiões Intermediárias do Estado do Pará estão assim constituídas:

a) Região intermediaria Altamira abrange 9 municípios: Almeirim, Altamira, Anapu, Brasil Novo, Medicilândia, Porto de Moz, Senador José Porfírio, Uruará e Vitória do Xingu.

b) Região Intermediária Belém abrange 23 municípios: Abaetetuba, Acará, Ananindeua, Barcarena, Belém, Benevides, Bujaru, Cametá, Colares, Concórdia do Pará, Igarapé-Miri, Limoeiro do Ajuru, Marituba, Mocajuba, Moju, Oeiras do Pará, Santa Bárbara do Pará, Santa Izabel do Pará, Santo Antônio do Tauá, São Caetano de Odivelas, Tailândia, Tomé-Açu e Vigia.

c) Região Intermediária Breves abrange 16 municípios: Afuá, Anajás, Bagre, Breves, Cachoeira do Arari, Chaves, Curralinho, Gurupá, Melgaço, Muaná, Ponta de Pedras, Portel, Salvaterra, Santa Cruz do Arari, São Sebastião da Boa Vista e Soure.

d) Região Intermediária Castanhal abrange 39 municípios: Augusto Corrêa, Aurora do Pará, Bonito, Bragança, Cachoeira do Piriá, Capanema, Capitão Poço, Castanhal, Curuçá, Dom Eliseu, Garrafão do Norte, Igarapé-Açu, Inhangapi, Ipixuna do Pará, Irituia, Mãe do Rio, Magalhães Barata, Maracanã, Marapanim, Nova Esperança do Piriá, Nova Timboteua, Ourém, Paragominas, Peixe-Boi, Primavera, Quatipuru, Salinópolis, Santa Luzia do Pará, Santa Maria do Pará, Santarém Novo, São Domingos do Capim, São Francisco do Pará, São João da Ponta, São João de Pirabas, São Miguel do Guamá, Terra Alta, Tracuateua, Ulianópolis e Viseu.

e) Região Intermediária Marabá abrange 23 municípios: Abel Figueiredo, Baião, Bom Jesus do Tocantins, Brejo Grande do Araguaia, Breu Branco, Canaã dos Carajás, Curionópolis, Eldorado do Carajás, Goianésia do Pará, Itupiranga, Jacundá, Marabá, Nova Ipixuna, Novo Repartimento, Pacajá, Palestina do Pará, Parauapebas, Piçarra, Rondon do Pará, São Domingos do Araguaia, São Geraldo do Araguaia, São João do Araguaia e Tucuruí.

f) Região Intermediária Redenção abrange 15 municípios: Água Azul do Norte, Bannach, Conceição do Araguaia, Cumaru do Norte, Floresta do Araguaia, Ourilândia do Norte, Pau D'Arco, Redenção, Rio Maria, Santa Maria das Barreiras, Santana do Araguaia, São Félix do Xingu, Sapucaia, Tucumã e Xinguara.

g) Região Intermediária Santarém abrange 19 municípios: Alenquer, Aveiro, Belterra, Curuá, Faro, Itaituba, Jacareacanga, Juruti, Mojuí dos Campos, Monte Alegre, Novo Progresso, Óbidos, Oriximiná, Placas, Prainha, Rurópolis, Santarém, Terra Santa e Trairão.

As variáveis estudadas foram os anos de ocorrência (2007, 2008, 2009, 2010, 2011, 2012, 2013, 2014, 2015 e 2016), a idade da criança em dias e anos ( 0 a 27 dias; 28 dias a <1 ano; 1 a 4 anos; 5 a 12 anos e Ignorado), diagnóstico final (sífilis congênita recente, sífilis congênita tardia, aborto por sífilis e natimorto por sífilis), faixa etária materna em $<20$ e $\geq 20$ anos e mais e Ignorado, escolaridade materna em anos de estudo (0 a $8, \geq 8$ e ignorada), cor da pele materna em branca e não branca, realização de pré-natal em Sim e Não, momento do diagnóstico da sífilis materna (pré-natal, parto/curetagem, Pós-parto, não realizado e ignorado), esquema de tratamento materno (adequado, inadequado, não realizado e ignorado) e número de óbitos por sífilis congênita. 
Por utilizar informações de acesso público este estudo dispensa o registro e avaliação no sistema CEP/CONEP, conforme a resolução o 510 de 07 de abril de 2016, pesquisas que utilizem informações de acesso público, nos termos da Lei $\mathrm{n} \cong 12.527$ de 18 de novembro de 2011.

Os dados foram processados e armazenados em banco estruturado utilizando-se o programa Microsoft Office Excel 2010. Foi utilizada estatística descritiva para avaliação de medidas de tendência central (média, mediana, desvio padrão), os testes $\mathrm{G}$ e Qui-quadrado para avaliação de associação, considerando-se o Intervalo de Confiança (IC) de 95\%, com significância estatística de $p \leq 0,05$.

\section{RESULTADOS}

No período de 2007 a 2016, foram diagnosticados e notificados 4710 casos de sífilis congênita, no Estado do Pará. A análise da sífilis congênita no período indica que o número de casos aumentou sistematicamente a cada ano, com pequeno decréscimo em 2012. Entretanto, a partir daí, percebe-se um aumento substancial na incidência da doença, apresentando em 2016 um número de casos superior a 2 vezes $(2,7)$ em relação ao ano de 2007. A análise dos dados por regiões geográficas mostra que Belém e Marabá foram as regiões do Estado com os maiores números de casos de sífilis congênita (Figura 1).

Figura 1 - Casos de sífilis congênita, por regiões geográficas intermediárias, no Pará - 2007 a 2016.

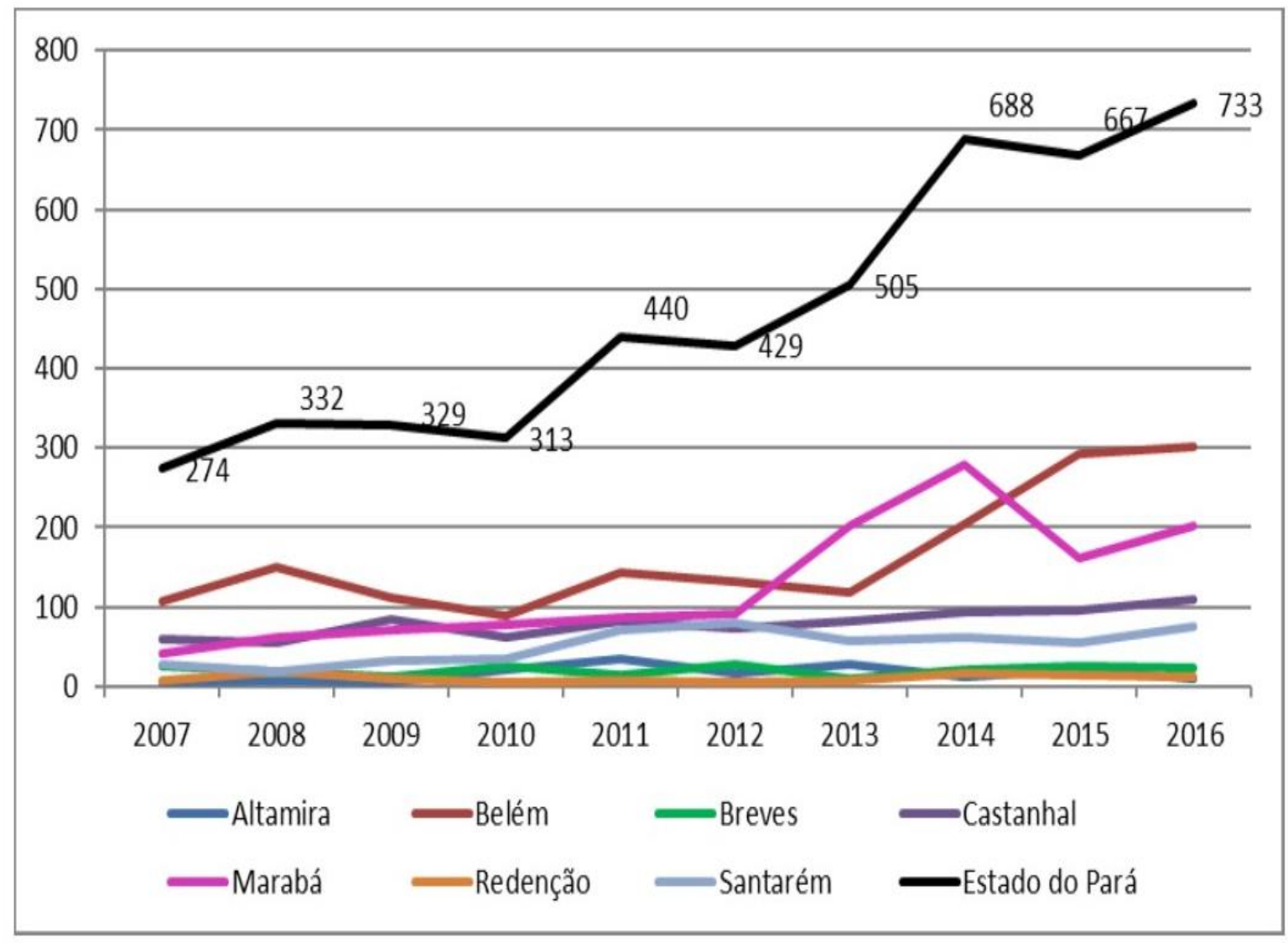

Fonte: Protocolo de pesquisa, 2018.

A taxa de incidência da sífilis congênita em menores de 1 ano de idade em 2007 quando comparada ao ano de 2016, aumentou em quase todas as regiões geográficas do Estado, excetuando-se a Região de Breves que engloba os municípios do Arquipélago do Marajó (Figura 2). 
Figura 2 - Taxa de incidência da sífilis congênita nas regiões geográficas do Pará, 2007 a 2016.

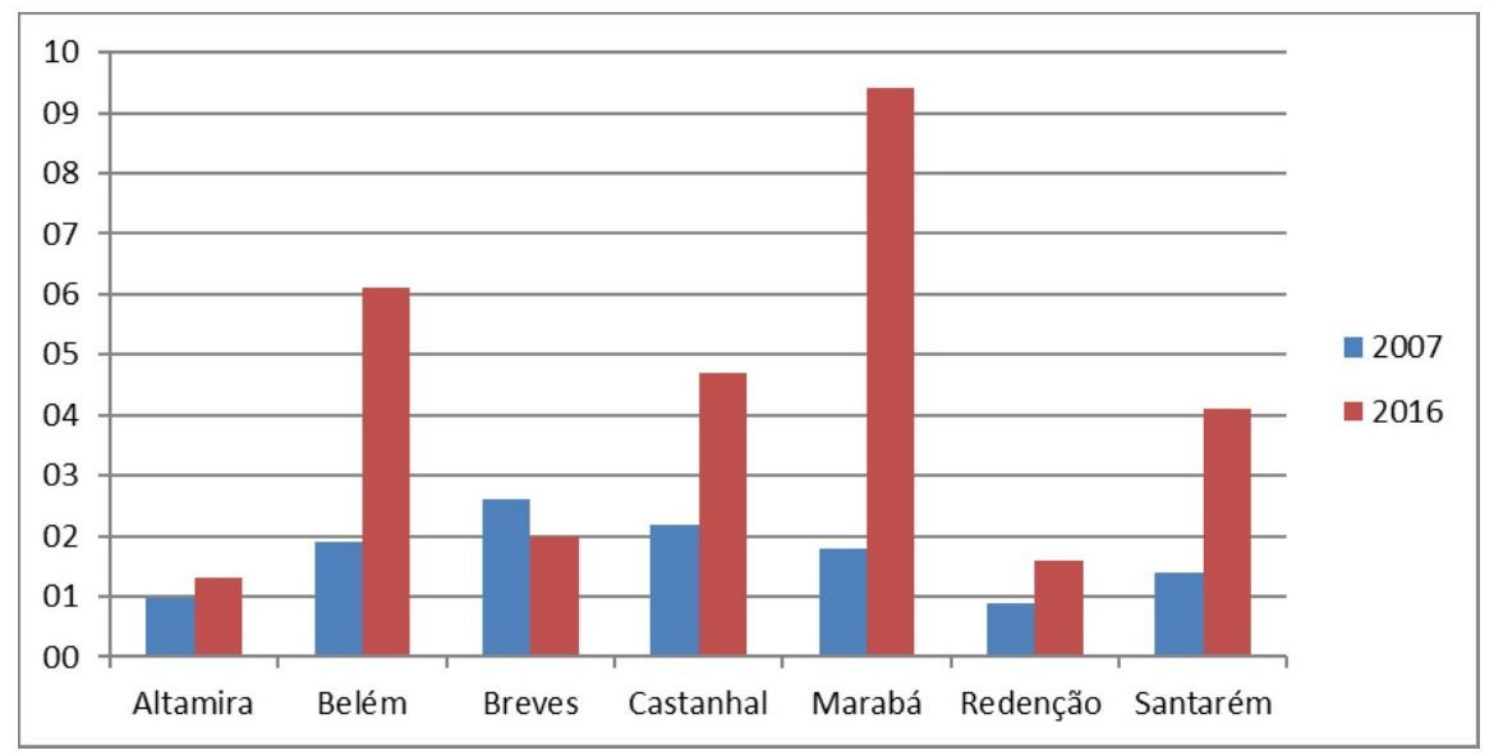

Fonte: Protocolo de pesquisa, 2018.

Ao analisar as características sociodemográficas, clínicas e de tratamento da sífilis congênita é observado que o diagnóstico da sífilis na criança ocorreu nos primeiros 27 dias de vida, sem diferença entre as regiões geográficas. Em relação às características maternas, é revelado que em todas as regiões do estado, as gestantes na grande maioria eram jovens, com idade superior a 20 anos, destacando-se Breves $(72,8 \%)$, Castanhal $(71,6 \%)$ e Marabá $(71,3 \%)$.

O pré-natal foi realizado pela grande maioria das gestantes, de forma significativa em todas as regiões, sobressaindo-se Altamira (90,1\%), Santarém (90,1\%), Marabá $(89,4 \%)$ e Castanhal $(82,9 \%)$. Entretanto, o momento do diagnóstico da sífilis se deu no parto e no pós-parto, tendo como exceção a região de Altamira. Assim, é possível registrar que $83,3 \%$ das gestantes com sífilis do Estado do Pará realizaram o pré-natal, excluindo-se daí os casos ignorados.

Em relação ao tratamento, observa-se que somente na região de Castanhal as gestantes não realizaram o tratamento para a sífilis ou este foi ignorado $(66,4 \%)$. Nas demais, o tratamento foi efetivado mesmo sendo a maioria de forma inadequada. Já seus parceiros não realizaram o tratamento ou este foi ignorado.

No que se refere ao diagnostico final, mais de $80,0 \%$ das crianças de todas as regiões do Estado tiveram o diagnóstico de sífilis recente.

Nesta mesma tabela é observado que o \% de realização do pré-natal foi expressiva. Apenas na região de Breves o percentual foi de $65,8 \%$; nas outras regiões o percentual foi maior que $77 \%$. Esse fato sugere a necessidade de melhorar a eficiência do Pré-natal.

Ainda podemos notar que nas 7 regiões, apenas em 2 (Breves e Castanhal) o diagnóstico não foi feito no pré-natal. O diagnóstico feito no pré-natal possibilitaria o tratamento da gestante, impedindo a SC.

O tratamento inadequado da mãe, que variou de $30 \%$ na região de Castanhal chegando a $76,4 \%$ na região de Altamira, justificaria a alta incidência de SC.

Vale registrar que Altamira foi a região com o maior percentual de gestante com idade menor de 20 anos (42,9\%). A escolaridade de até 8 anos de estudo predominou em seis regiões do Estado, chamando a atenção a região de Breves, com 70,3\%. A região de Belém, que engloba a capital do Estado, foi a que registrou 0 menor percentual de baixa escolaridade, e também o maior percentual de escolaridade ignorada $(39,9 \%)$. A cor da pele não branca foi a mais frequente em todas as regiões (Tabela 1). 
Tabela 1 - Características sociodemográficas, clínicas e de tratamento da sífilis congênita no Estado do Pará-Brasil, por regiões geográficas intermediárias. 2007 a 2016.

\begin{tabular}{|c|c|c|c|c|c|c|c|c|}
\hline \multirow[b]{2}{*}{ Características } & \multicolumn{7}{|c|}{ Regiões Geográficas Intermediárias } & \multirow[t]{2}{*}{$p$} \\
\hline & $\begin{array}{c}\text { Altamira } \\
\begin{array}{c}\mathrm{N}=161 \\
\mathrm{~N}(\%)\end{array}\end{array}$ & $\begin{array}{c}\text { Belém } \\
\text { N= } 1644 \\
N(\%)\end{array}$ & $\begin{array}{c}\text { Breves } \\
N=202 \\
N(\%)\end{array}$ & $\begin{array}{c}\text { Castanhal } \\
\begin{array}{c}\mathrm{N}=797 \\
\mathrm{~N}(\%)\end{array}\end{array}$ & $\begin{array}{c}\text { Marabá } \\
\text { N= } 1277 \\
N(\%)\end{array}$ & $\begin{array}{c}\text { Redenção } \\
\begin{array}{c}\mathrm{N}=105 \\
\mathrm{~N}(\%)\end{array}\end{array}$ & $\begin{array}{c}\text { Santarém } \\
\begin{array}{c}\mathrm{N}=516 \\
\mathrm{~N}(\%)\end{array}\end{array}$ & \\
\hline \multicolumn{9}{|l|}{ Idade da Criança } \\
\hline 0 a $27 d$ & $157(97,5)$ & $1600(97,3)$ & $200(99,0)$ & $781(98,0)$ & $1260(98,7)$ & $95(90,5)$ & $499(96,7)$ & $0,0070^{b}$ \\
\hline $28 \mathrm{~d}$ a $11 \mathrm{~m}$ & $3(1,9)$ & $35(2,1)$ & $2(1,0)$ & $14(1,8)$ & $17(1,3)$ & $8(7,6)$ & $15(2,9)$ & \\
\hline 1 a 4 anos & $1(0,6)$ & $7(0,5)$ & $0(0,0)$ & $1(0,1)$ & $0(0,0)$ & $2(1,9)$ & $1(0,2)$ & \\
\hline 5 a 12 anos & $0(0,0)$ & $2(0,1)$ & $0(0,0)$ & $1(0,1)$ & $0(0,0)$ & $0(0,0)$ & $1(0,2)$ & \\
\hline Ignorado & $0(0,0)$ & $0(0,0)$ & $0(0,0)$ & $0(0,0)$ & $0(0,0)$ & $0(0,0)$ & $0(0,0)$ & \\
\hline \multicolumn{9}{|l|}{ Faixa etária } \\
\hline$<20$ anos & $69(42,9)$ & $515(31,3)$ & $53(26,2)$ & $206(25,9)$ & $348(27,2)$ & $39(37,1)$ & $166(32,2)$ & $<0,0001^{c}$ \\
\hline$\geq 20$ anos & $91(56,5)$ & $1103(67,1)$ & $147(72,8)$ & $571(71,6)$ & $910(71,3)$ & $59(56,2)$ & $348(67,4)$ & \\
\hline Ignorada & $1(0,6)$ & $26(1,6)$ & $2(1,0)$ & $20(2,5)$ & $19(1,5)$ & $7(6,7)$ & $2(0,4)$ & \\
\hline \multicolumn{9}{|l|}{ Escolaridade } \\
\hline$\leq 8$ & $88(54,6)$ & $707(43,0)$ & $142(70,3)$ & $470(59,0)$ & $818(64,1)$ & $68(64,7)$ & $350(67,8)$ & $<0,0001^{\mathrm{c}}$ \\
\hline$>8$ & $32(19,9)$ & $281(17,1)$ & $23(11,4)$ & $127(15,9)$ & $329(25,7)$ & $11(10,5)$ & $129(25,0)$ & \\
\hline Ignorada & $41(25,5)$ & $656(39,9)$ & $37(18,3)$ & $200(25,1)$ & $130(10,2)$ & $26(24,8)$ & $37(7,2)$ & \\
\hline \multicolumn{9}{|l|}{ Cor da pele } \\
\hline Branca & $16(10,0)$ & $113(6,9)$ & $19(9,4)$ & $39(4,9)$ & $103(8,1)$ & $15(14,3)$ & $41(7,9)$ & $0,0076^{c}$ \\
\hline Não Branca & $143(88,8)$ & $1386(84,3)$ & $177(87,6)$ & $741(93,0)$ & $1162(91,0)$ & $89(84,8)$ & $471(91,3)$ & \\
\hline Ignorada & $2(1,2)$ & $145(8,8)$ & $6(3,0)$ & $17(2,1)$ & $12(0,9)$ & $1(0,9)$ & $4(0,8)$ & \\
\hline
\end{tabular}




\begin{tabular}{|c|c|c|c|c|c|c|c|c|}
\hline \multicolumn{9}{|l|}{ Pré-Natal } \\
\hline Sim & $145(90,1)$ & $1289(78,4)$ & $133(65,8)$ & $661(82,9)$ & $1142(89,4)$ & $81(77,1)$ & $465(90,1)$ & $<0.0001^{\mathrm{c}}$ \\
\hline Não & $16(9,9)$ & $305(18,6)$ & $65(32,2)$ & $109(13,7)$ & $105(8,2)$ & $11(10,5)$ & $41(8,0)$ & \\
\hline Ignorado & $0(0,0)$ & $50(3,0)$ & $4(2,0)$ & $27(3,4)$ & $30(2,4)$ & $13(12,4)$ & $10(1,9)$ & \\
\hline \multicolumn{9}{|c|}{ Momento do Diagnóstico } \\
\hline Pré-natal & $80(49,7)$ & $602(36,6)$ & $59(29,2)$ & $237(29,8)$ & $635(49,7)$ & $48(45,7)$ & $254(49,2)$ & $<0,0001^{b}$ \\
\hline Parto & $16(10,0)$ & $540(32,8)$ & $53(26,2)$ & $366(45,9)$ & $478(37,4)$ & $24(22,9)$ & $73(14,1)$ & \\
\hline Pós-Parto & $63(39,1)$ & $409(24,9)$ & $82(40,6)$ & $147(18,4)$ & $121(9,5)$ & $20(19,0)$ & $159(30,8)$ & \\
\hline Não realizou & $0(0,0)$ & $13(0,8)$ & $4(2,0)$ & $18(2,3)$ & $12(1,0)$ & $2(1,9)$ & $6(1,2)$ & \\
\hline Ignorado & $2(1,2)$ & $80(4,9)$ & $4(2,0)$ & $29(3,6)$ & $31(2,4)$ & $11(10,5)$ & $24(4,7)$ & \\
\hline \multicolumn{9}{|l|}{ Tratamento mãe } \\
\hline Adequado & $20(12,4)$ & $110(6,7)$ & $22(10,9)$ & $29(3,6)$ & $146(11,4)$ & $16(15,2)$ & $131(25,4)$ & $<0,0001^{\mathrm{c}}$ \\
\hline Inadequado & $123(76,4)$ & $849(51,7)$ & $125(61,9)$ & $239(30,0)$ & $858(67,2)$ & $39(37,1)$ & $228(44,2)$ & \\
\hline Não realizou & $9(5,6)$ & $507(30,8)$ & $39(19,3)$ & $385(48,3)$ & $224(17,6)$ & $28(26,7)$ & $90(17,4)$ & \\
\hline Ignorado & $9(5,6)$ & $178(10,8)$ & $16(7,9)$ & $144(18,1)$ & $49(3,8)$ & $22(21,0)$ & $67(13,0)$ & \\
\hline \multicolumn{9}{|c|}{ Tratamento Parceiro } \\
\hline Sim & $36(22,4)$ & $261(15,9)$ & $47(23,3)$ & $95(12,0)$ & $288(22,6)$ & $23(21,9)$ & $168(32,6)$ & $<0,0001^{\mathrm{c}}$ \\
\hline Não & $105(65,2)$ & $1011(61,5)$ & $119(58,9)$ & $504(63,2)$ & $874(68,4)$ & $48(45,7)$ & $265(51,3)$ & \\
\hline Ignorado & $20(12,4)$ & $372(22,6)$ & $36(17,8)$ & $198(24,8)$ & $115(9,0)$ & $34(32,4)$ & $83(16,1)$ & \\
\hline \multicolumn{9}{|c|}{ Diagnóstico Final } \\
\hline Recente & $156(97,0)$ & $1463(89,0)$ & $189(93,5)$ & $741(93,0)$ & $1225(95,9)$ & $93(88,6)$ & $484(93,8)$ & $<0,0001^{b}$ \\
\hline Tardia & $1(0,6)$ & $12(0,7)$ & $7(3,5)$ & $3(0,4)$ & $1(0,1)$ & $0(0,0)$ & $2(0,4)$ & \\
\hline Aborto & $2(1,2)$ & $68(4,1)$ & $0(0,0)$ & $5(0,6)$ & $17(1,3)$ & $2(1,9)$ & $2(0,4)$ & \\
\hline Natimorto & $2(1,2)$ & $101(6,2)$ & $6(3,0)$ & $48(6,0)$ & $34(2,7)$ & $10(9,5)$ & $28(5,4)$ & \\
\hline
\end{tabular}

Fonte: Protocolo de pesquisa, 2018. a Na análise estatística foram excluídos os dados relativos aos ignorados, b Teste G; c Teste X. 
A ausência de tratamento do parceiro variou de $45,7 \%$ na região de Redenção a $68,4 \%$ na região de Marabá, sugerindo que a reinfecção da gestante poderia estar associada ao insucesso do pré-natal na prevenção da SC.

As variáveis sociodemográficas que se associaram $(p<0,05)$ com a ocorrência de sífilis congênita, no Estado, foram a escolaridade materna $\leq 8$ anos de estudo ( $R R=1,06$; IC $95 \% 1,00-1,25)$; cor da pele não branca (RR=0,71; IC95\% 0,66-0,77), conforme Tabela 2.

Tabela 2 - Características sociodemográficas associadas à ocorrência de Sífilis congênita - Pará 2007 a 2016.

\begin{tabular}{|c|c|c|c|c|c|}
\hline \multirow[t]{2}{*}{ Características } & \multirow[b]{2}{*}{$\mathrm{N}^{\mathrm{a}}$} & \multicolumn{2}{|c|}{ Sífilis congênita } & \multirow{2}{*}{$\begin{array}{c}\mathbf{R R}^{\mathbf{b}} \\
\left(\mathrm{IC}_{95 \%}\right)^{\mathrm{C}}\end{array}$} & \multirow[b]{2}{*}{ Valor de $p$} \\
\hline & & $\mathrm{n}^{\mathrm{a}}$ & Incidência (\%) & & \\
\hline \multicolumn{6}{|l|}{ Idade } \\
\hline$<20$ & 2843 & 1396 & 49,1 & $0,98(0,94-1,03)$ & 0,2193 \\
\hline$\geq 20$ & 6458 & 3229 & 50,0 & & \\
\hline \multicolumn{6}{|l|}{ Escolaridade } \\
\hline$\leq 8$ & 5407 & 2643 & 48,9 & $1,06(1,00-1,25)$ & 0,0224 \\
\hline$>8$ & 2016 & 932 & 46,2 & & \\
\hline \multicolumn{6}{|l|}{ Cor da pele } \\
\hline Branca & 699 & 346 & 49,5 & $0,71(0,66-0,77)$ & $<0,0001$ \\
\hline Não Branca & 6012 & 4169 & 69,3 & & \\
\hline
\end{tabular}

Fonte: Protocolo de pesquisa, 2018. ${ }^{\text {a }}$ Totais excluídos os dados ignorados; ${ }^{b}$ RR: Risco Relativo; ${ }^{\mathrm{c}} \mathrm{IC}_{95 \%}$ : Intervalo de Confiança.

\section{DISCUSSÃO}

Os resultados deste estudo mostram que no Estado do Pará, tanto o número dos casos de sífilis congênita quanto a taxa de incidência por mil nascidos vivos apresentaram tendência de aumento no período 2007 a 2016, de cerca de 3 vezes. A taxa de incidência de sífilis congênita que era de 1,8 em 2007 passou para 5,3 em 2016, acompanhando o aumento da taxa de incidência ocorrida no País, que no mesmo período apresentou um aumento de 3,6 vezes (BRASIL, 2017).

As regiões geográficas que mais contribuíram para esse aumento foram Belém, Marabá e Castanhal. 0 aumento da incidência aqui identificado pode ser atribuído a inúmeros fatores, como à melhoria da notificação dos casos ao longo dos anos, à dispensação dos testes rápidos nas unidades municipais de saúde, aos avanços da vigilância epidemiológica e à capacitação dos profissionais da saúde. Por outro lado, esses dados podem também apontar uma baixa qualidade da assistência materno infantil e a dificuldade em se estabelecer o diagnóstico precoce no pré-natal e realização do tratamento adequado dos casos positivos.

Uma atenção ao pré-natal e puerperal qualificada e humanizada se dá por meio da incorporação de condutas acolhedoras e humanizadas, garantindo a captação precoce das gestantes com realização da primeira consulta de pré-natal até 120 dias da gestação, num total de seis consultas de pré-natal, sendo, preferencialmente, uma no primeiro trimestre, duas no segundo trimestre e três no terceiro trimestre da gestação (BRASIL, 2006).

Segundo Kroeger KA, et al. (2018) para a prevenção da SC, uma mulher precisa de diagnóstico oportuno da gravidez e do início do pré-natal, um número adequado de consultas de pré-natal durante a gravidez e triagem para sífilis em intervalos apropriados durante a gravidez e no parto. Caso diagnosticada com sífilis deverá ser tratada com penicilina benzatina não menos de 30 dias antes do parto.

A sífilis congênita é uma doença evitável e sua ocorrência não deve ser tolerada, pois até mesmo um caso representa uma falha do sistema público de saúde retratada pelo aumento das intercorrências incluem prematuridade, natimorto, hidropisia fetal não imune e mortalidade neonatal (COOPER JM, et al., 2016). 
Neste estudo, a sífilis congênita foi mais incidente em crianças nascidas de mulheres entre 20 anos ou mais de idade (68,7\%), de cor não branca e com baixa escolaridade, indicando maior vulnerabilidade social desta população. Esses achados podem ser explicados pelo fato de que é nessa faixa de idade que a mulher exerce sua plena sexualidade e, portanto, com maiores probabilidades de ocorrência de uma gravidez. A baixa escolaridade e idade maior de 20 anos são características frequentes em mulheres com sífilis. A baixa escolaridade e idade maior de 20 anos são características frequentes em mulheres com sífilis. Resultados semelhantes foram encontrados por inúmeros estudos, corroborando os presentes achados (LAFETA KR, et al., 2016; NONATO SM, et al. 2015; COSTA CC, et al. 2013; ALMEIDA MFG, PEREIRA SM, 2007).

Sabe-se que a baixa escolaridade pode se constituir em fator limitante ao acesso às informações indispensáveis ao autocuidado, à conscientização sobre a saúde sexual e às medidas de prevenção da doença. Daí que o maior desafio é garantir que as gestantes possam se beneficiar do tratamento da sífilis e prevenir a sífilis congênita. Merece atenção referir que cerca de um terço das crianças com sífilis congênita eram de mulheres menores de 20 anos, período de maior risco para as práticas sexuais sem a adoção de medidas de proteção tanto para as doenças sexualmente transmissíveis (DST), quanto para a gravidez (ROKHMAH D, KHOIRON, 2015).

Observou-se que a maioria das mulheres deste estudo, independente das regiões geográficas do Estado, realizou o pré-natal, porém o momento do diagnóstico da sífilis congênita ocorreu em grande parte (44,9\%) somente por ocasião do parto e pós-parto, evidenciando a falha da assistência ao pré-natal e do controle de infecções sexualmente transmissíveis. Dados semelhantes sobre a realização do pré-natal foram encontrados por Costa CC, et al. (2013) e Teixeira LO et al. (2018) estudando a sífilis congênita no Ceará e no estado do Rio Grande do Sul, respectivamente.

Segundo Phiske MM (2014), a pedra angular do controle da sífilis congênita é a triagem pré-natal e o tratamento de mães, daí a necessidade de seu fortalecimento, principalmente nos países em desenvolvimento. Os dados deste estudo corroboram com essa premissa, visto que 2012 e 2016 foram os anos com o maior número de atendimento pré-natal e também o de maior número de casos de SC diagnosticados.

A partir do diagnóstico deve-se instituir de forma imediata o tratamento adequado tanto para as gestantes, quanto para seus parceiros e de acordo com o Ministério da Saúde, o tratamento é considerado adequado, quando é completo, adequado ao estágio da doença, feito com penicilina e finalizado pelo menos 30 dias antes do parto, tendo sido o parceiro tratado concomitantemente (BRASIL, 2018; WHO, 2016). Neste estudo, a maioria das gestantes realizou tratamento inadequado e seus parceiros não foram tratados. Fato que possivelmente explica o aumento dos casos de sífilis congênita no Estado do Pará.

Cabe mencionar que o presente estudo apresenta limitações relacionadas à qualidade da informação disponível no SINAN como a ausência de informações sociodemográficas, ocasionando dificuldades na identificação do perfil social da população estudada. Outra limitação que merece atenção dos gestores responsáveis é o grande número de registro de informações ignoradas, o que impossibilita uma análise mais fidedigna do perfil epidemiológico da sífilis congênita no Estado do Pará e consequentemente no Brasil. Entretanto, a utilização de sistemas de informação tem sido considerada uma valiosa estratégia para análises epidemiológicas; considerando sua importância para a tomada de decisão dos gestores e sua abrangência territorial (LIMA CRA, et al. 2009).

\section{CONCLUSÃO}

Os resultados deste estudo evidenciam um aumento na incidência da SC em mulheres maiores de 20 anos, em todas as regiões geográficas do estado do Pará. Dados estes que evidenciam a baixa qualidade da assistência no pré-natal, falha na prevenção da sífilis congênita, diagnóstico tardio da sífilis (no pós-parto) e tratamento inadequado da gestante e seu parceiro, expressados na alta incidência da SC encontrada no período estudado. 


\section{REFERÊNCIAS}

1. ALMEIDA MFG, PEREIRA SM. Caracterização epidemiológica da sífilis congênita no município de Salvador, Bahia. DST- J. Bras. Doenças Sex. Transm. 2007; 19(3-4): 144-156.

2. BRASIL. Ministério da Saúde. Sífilis. Disponível em: http://www.aids.gov.br/pt-br/publico-geral/o-que-sao-ist/sifilis. Acesso em: 12 mai. 2018

3. BRASIL, Ministério da Saúde Secretaria de Vigilância em Saúde, Departamento de DST, Aids e Hepatites Virais. Sífilis 2017. Boletim Epidemiológico. 2017; 48 (36). Disponível em: http://www.aids.gov.br/pt-br/pub/2017/boletimepidemiologico-de-sifilis-2017. Acesso em: 14 abr. 2018.

4. BRASIL Ministério da Saúde. Secretaria de Vigilância em Saúde. Programa Nacional de DST/AIDS. Diretrizes para controle da sífilis congênita: manual de bolso / Ministério da Saúde, Secretaria de Vigilância em Saúde, Programa Nacional de DST/Aids. - 2. ed. - Brasília: Ministério da Saúde, 2006. 72 p. il. - (Série Manuais 24).

5. COOPER JM, MICHELOW IC, WOZNIAK PS, et al. In time: the persistence of congenital syphilis in Brazil-more progress needed! Rev Paulista Pediatr. 2016;34(3):251-253.

6. COSTA CC, FREITAS LV, SOUSA DMN et al. Sífilis congênita no Ceará: análise de uma década. Rev Esc Enferm USP. 2013; 47(1):152-9.

7. INSTITUTO BRASILEIRO DE GEOGRAFIA E ESTATÍSTICA. IBGE. Panorama do Estado do Pará. Disponível em: https://cidades.ibge.gov.br/brasil/pa/panorama. Acesso em: 17 mai. 2019.

8. INSTITUTO BRASILEIRO DE GEOGRAFIA E ESTATÍSTICA. IBGE. Divisão regional do Brasil em regiões geográficas imediatas e regiões geográficas intermediárias: 2017 / IBGE, Coordenação de Geografia. - Rio de Janeiro: IBGE, 2017. 82p.

9. KROEGER KA, SANGARAMOORTHY T, LOOSIER PS, ET AL. Pathways to Congenital Syphilis Prevention: A Rapid Qualitative Assessment of Barriers, and the Public Health Response, in Caddo Parish, Louisiana. Rev Sexually Transmitted Diseases, 2018; 45 (7): 442-446.

10. LAFETA, K.R.G; MARTELLI JUNIOR, H; SILVEIRA, M.F, et al. Sífilis materna e congênita, subnotificação e difícil controle. Revista Brasileira de Epidemiologia, 2016; 19(1): 63-74.

11. LIMA CRA, SCHRAMM JMA, COELI, CM et al. Revisão das dimensões de qualidade dos dados e métodos aplicados na avaliação dos sistemas de informação em saúde. Cad. Saúde Pública. 2009; 25(10): 2095-2109.

12. NONATO, S. M.; MELO, A. P. S; GUIMARÃES, M. D.C. Sífilis na gravidez e fatores associados à sífilis congênita em Belo Horizonte MG, 2010-2013. Epidemiol. Serv Saúde[online]. 2015; 24 (4): 681-94.

13. PHISKE, M.M. Current trends in congenital syphilis. Indian J Sex Transm Dis. 2014; 35(1):12-20.

14. ROKHMAH D, KHOIRON. The Role of Sexual Behavior In The Transmission Of HIV and AIDS In Adolescent In Coastal Area (International Conference on Tropical and Coastal Region Eco-Development 2014). Procedia Environmental Sciences. 2015; 23:99 - 104.

15. SARACENI V, PEREIRA GFM, SILVEIRA MF, et al. Vigilância epidemiológica da transmissão vertical da sífilis: dados de seis unidades federativas no Brasil. Rev Panam Salud Publica, 41:e44, 2017.

16. TEIXEIRA LO, BELARMINIO V, GONÇALVES CV, et al. Tendência temporal e distribuição espacial da sífilis congênita no estado do Rio Grande do Sul entre 2001 e 2012. Ciência \& Saúde Coletiva. 2018; 23(8): $2587-97$.

17. WORLD HEALTH ORGANIZATION. WHO. Global health sector strategy on sexually transmitted infections $2016-$ 2021. Geneva, Switzerland, 2016; 64p.

18. World Health Organization. WHO. Who Sexually transmitted infections health topic. Disponível em: https://www.who.int/news-room/fact-sheets/detail/sexually-transmitted-infections-(stis). Acesso em: 13 jun.2019. 